\title{
Research
}

\section{Alpha and beta diversity of connected benthic-subsurface invertebrate communities respond to drying in dynamic river ecosystems}

\author{
Rachel Stubbington, Romain Sarremejane and Thibault Datry
}

R. Stubbington (https://orcid.org/0000-0001-8475-5109) $\square$ (rachel.stubbington@ntu.ac.uk) and R. Sarremejane, School of Science and Technology, Nottingham Trent Univ., Nottingham, UK. - T. Datry, IRSTEA, UR RiverLY, Centre de Lyon-Villeurbanne, France.

\section{Ecography}

42: 2060-2073, 2019

doi: $10.1111 /$ ecog. 04592

Subject Editor: Jason Pither Editor-in-Chief: Miguel Araújo Accepted 15 August 2019
Drying disturbances are the primary determinant of aquatic community biodiversity in dynamic river ecosystems. Research exploring how communities respond to disturbance has focused on benthic invertebrates in surface sediments, inadequately representing a connected community that extends into the subsurface. We compared subsurface and benthic invertebrate responses to drying, to identify common and context-dependent spatial patterns. We characterized community composition, alpha diversity and beta diversity across a gradient of drying duration. Subsurface communities responded to drying, but these responses were typically less pronounced than those of benthic communities. Despite compositional changes and in contrast to reductions in benthic alpha diversity, the alpha diversity of subsurface communities remained stable except at long drying durations. Some primarily benthic taxa were among those whose subsurface frequency and abundance responded positively to drying. Collectively, changing composition, stable richness and taxon-specific increases in occurrence provide evidence that subsurface sediments can support persistence of invertebrate communities during drying disturbances. Beta-diversity patterns varied and no consistent patterns distinguished the total diversity, turnover or nestedness of subsurface compared to benthic communities. In response to increasing drying duration, beta diversity increased or remained stable for benthic communities, but remained stable or decreased for subsurface communities, likely reflecting contrasts in the influence of mass effects, priority effects and environmental filtering. Dissimilarity between subsurface and benthic communities remained stable or increased with drying duration, suggesting that subsurface communities maintain distinct biodiversity value while also supporting temporary influxes of benthic taxa during drying events. As temporary rivers increase in extent due to global change, we highlight that recognizing the connected communities that extend into the subsurface sediments can enable holistic understanding of ecological responses to drying, the key determinant of biodiversity in these dynamic ecosystems.

Keywords: $\alpha$-diversity, $\beta$-diversity, hyporheic zone, intermittent rivers and ephemeral streams (IRES), macroinvertebrates, refuge 


\section{Introduction}

Understanding how environmental factors influence spatial biodiversity patterns is a central goal in ecology. Interactions between alpha $(\alpha)$ and beta $(\beta)$ diversities determine regionalscale patterns, and their concurrent study underpins understanding of communities within a metacommunity context (Baselga 2010, Tuomisto 2010). In dynamic ecosystems, disturbances (sensu Townsend 1989) are fundamental forces that alter physical environments to influence how biodiversity is organized in space and time (Huston 1979, Pickett et al. 1989). Many studies have characterized ecological responses to disturbance within regions. However, we need to better understand how communities respond at large spatial scales, to identify context-independent relationships and inform development of general 'rules' that explain how ecosystems function. Equally, identifying context-dependent relationships can underpin effective management that supports biodiverse communities within regions.

Community composition varies in response to disturbance intensity (Lepori and Malmqvist 2009, Vanschoenwinkel et al. 2010), with lower $\alpha$-diversity (i.e. local community diversity within a sampling unit; Tuomisto 2010) often reflecting environmental instability at highly disturbed sites (Denslow 1980, Datry et al. 2014a). Communities at more disturbed sites may be dominated by generalists that represent 'nested' subsets of the taxa also found at less disturbed sites (Fernández-Juricic 2002, Datry et al. 2014a). Alternatively, habitat heterogeneity (i.e. the range of habitat types present at a site; Szewczyk and McCain 2019) at disturbed sites can create niches in which adapted specialists replace taxa restricted to less disturbed sites, offsetting biodiversity declines (Wesche et al. 2000, Bogan et al. 2013). Taxa losses/ gains (nestedness) and replacements (turnover) influence how $\beta$-diversity (i.e. community variation among sampling units; Anderson et al. 2011) responds to disturbance intensity in dynamic ecosystems (Aspin et al. 2018). Beta-diversity may peak at stable sites if environmental filtering homogenizes communities at disturbed sites (Chase 2007, Lepori and Malmqvist 2009). Alternatively, habitat heterogeneity may allow $\beta$-diversity to peak at intermediate disturbance intensities (Grime 1973, Cadotte 2007, Leigh and Datry 2017), if community homogenization is restricted to highly disturbed sites (Chase 2007) and mass effects allow taxa with high dispersal rates to reduce community variability at stable sites (Heino et al. 2015).

Rivers are dynamic ecosystems in which floods and drying (i.e. extensive or complete loss of water from the surface and sometimes subsurface sediments) are common disturbances, and drying is natural and often predictable in temporary rivers. The connectivity of riverbed sediments creates extensive habitats in which organisms move between surface (i.e. benthic) and subsurface (i.e. hyporheic) sediments in response to environmental drivers. The subsurface sediments can be an ecological refuge during disturbances (Davis et al. 2013), supporting the persistence of connected communities that span the habitats within a river's vertical dimension (Stubbington 2012). In dynamic ecosystems, such refuges can mediate responses to disturbance and stabilize populations (Berryman et al. 2006), but most ecohydrological studies have considered only benthic communities within surface sediments, without recognizing that communities extend into the subsurface.

Based on macroinvertebrate community characterizations, subsurface and benthic communities differ, with lower $\alpha$ - and $\beta$-diversity of subsurface communities attributed to their inhabitation of a relatively homogeneous environment (Datry 2012). These connected communities may therefore respond differently to disturbance, with lower disturbance intensities and $\alpha$-diversity sometimes reducing subsurface response magnitudes (Young et al. 2011, Datry 2012). Lower disturbance magnitudes can allow subsurface sediments to provide a refuge for benthic organisms (Clinton et al. 1996, Vander Vorste et al. 2016), and benthic responses to disturbance may therefore influence changes in subsurface communities (Stubbington 2012) and alter similarity between these interacting faunas. In temporary rivers, subsurface $\alpha$-diversity may decrease in response to drying if declining water availability reduces survival of sensitive taxa (Stanley et al. 1994, Clinton et al. 1996). Alternatively, $\alpha$-diversity may remain stable or increase if surface water loss triggers vertical migration of benthic organisms into saturated or humid subsurface sediments (Clinton et al. 1996, Vander Vorste et al. 2016). The arrival of benthic migrants may increase both total similarity and the nestedness contribution to similarity between subsurface and benthic communities. Across the vertical dimension, divergent niche selection in heterogeneous environments may increase $\beta$-diversity at temporary compared to perennial sites (Young et al. 2011, Stubbington et al. 2017a). Subsurface $\beta$-diversity may respond to any increase in the occurrence of predominantly benthic taxa by increasing, or by decreasing if a common subset of taxa joins variable subsurface communities.

Whereas the profound influence of drying on $\alpha$-diversity and, to a lesser extent, $\beta$-diversity is well-characterized for benthic invertebrate communities during flowing phases (Datry et al. 2014a, Leigh and Datry 2017), how drying affects subsurface communities and their interactions with benthic organisms remains poorly known (but see Clinton et al. 1996, Datry et al. 2007, Stubbington et al. 2011b, Datry 2012), and no study has described large-scale spatial patterns. To address this research gap, we compiled all available data documenting subsurface-benthic communities in perennial and temporary rivers. Our aim was to quantify responses of invertebrate communities in subsurface sediments to surface water loss, and to compare these to benthic community responses.

Our first hypothesis was that subsurface community composition changes in response to drying, but that this response is reduced compared to benthic communities due to lower disturbance magnitude and lower total $\alpha$-diversity. Second, we hypothesized that $\alpha$-diversity decreases in response 
to drying duration due to the loss of drying-sensitive organisms, but that the gain of primarily benthic taxa may offset or exceed the loss of sensitive invertebrates from subsurface sediments at temporary sites with greater water permanence; again, we hypothesized that any subsurface decrease would be reduced compared to benthic communities (Fig. 1a). Third, we hypothesized that $\beta$-diversity is lower in subsurface compared to benthic communities, due to lower habitat diversity and accessibility. We also hypothesized that the $\beta$-diversity of both communities increases with decreasing water permanence up to a threshold (due to greater environmental variability), then declines at longer drying durations, with more pronounced changes in subsurface communities joined by benthic migrants (Fig. 1b). Our fourth hypothesis was that with decreasing water permanence, total similarity and nestedness between subsurface and benthic communities increase, due to greater use of subsurface sediments by predominantly benthic taxa (Fig. 1c).

\section{Material and methods}

\section{Data sets}

We sought data comprising subsurface and benthic aquatic invertebrate communities sampled concurrently during flowing phases at $\geq 3$ sites on one river with variation in water permanence. We sourced data from 55 sites across five rivers (Table 1), which (based on a comprehensive literature search and correspondence with experts) we believe represent all globally available data. Two rivers are in France (Albarine, Asse), two in the UK (Glen, Lathkill) and one in New Zealand (Selwyn), the latter characterized by an invertebrate fauna distinct from that in European rivers (Supplementary material Appendix 2 Table A3-A5). All rivers are in temperate climate regions, including four oceanic $(\mathrm{Cfb})$ and one mediterranean (Csa) region (Köppen-Geiger classification; Table 1). All studies collected benthic samples by manually disturbing sediments to a depth of approx. $5 \mathrm{~cm}$ within an area of approx. $0.1 \mathrm{~m}^{2}$, and collected subsurface samples by pumping 4-61 of water and associated organisms from a depth of $30 \mathrm{~cm}$ below the bed; further details are provided in the Supplementary material Appendix 1.

\section{Water permanence regimes}

At each site on each river, water permanence in the surface stream was quantified or estimated for the two years preceding the first invertebrate sample collection date, and stated as the \% of the period without surface water (hereafter, $\%$ time dry). Datry et al. (2014a) describe calculation of $\%$ time dry in the Albarine, Asse and Selwyn. For the Glen and Lathkill, estimates were informed by long-term gauging station data, information from local hydrologists, our own on-site observations and meteorological data (Met Office 2019). Water permanence was particularly variable among sites on the alluvial rivers Selwyn (0-89\%; Larned et al. 2008) and Albarine (0-60\%; Datry et al. 2014b), with 0\% and $89 \%$ indicating sites which never dry (i.e. perennial sites) and which dry for approx. $325 \mathrm{~d} \mathrm{yr}^{-1}$, respectively (Table 1). In contrast, temporary sites on the Asse, Glen and Lathkill dried for $<90 \mathrm{~d} \mathrm{yr}^{-1}$ in the two years preceding first sample collection.

No study quantified the extent to which the water table declined below the sediment surface, although water loss from sampled subsurface sediments occurred within seven days of surface water loss in the Albarine (Datry 2012) and our site-specific observations indicate comparable loss rates in other rivers. All rivers are characterized by predictable changes in water permanence in response to seasonal variation in the water table (Datry et al. 2007, 2014b, Stubbington et al. 2011a, b, Datry 2012). Above-average rainfall (Met Office 2019) prevented typical annual drying in the Glen and Lathkill during the study period, and samples were therefore collected after approx. 8-12 months of continuous flow at temporary sites, interrupted only by two short dry periods at one Glen site (Stubbington et al. 2011b).

\section{Invertebrate data}

For each river, we created three data sets: subsurface samples, benthic samples and subsurface-benthic sample 'pairs'. Subsurface and benthic samples were equal in number and collected concurrently in the Albarine, Asse, Glen and Lathkill. In the Selwyn, subsurface and benthic samples collected within two days of each other were used to create 43 sample 'pairs', with one subsurface sample (a)

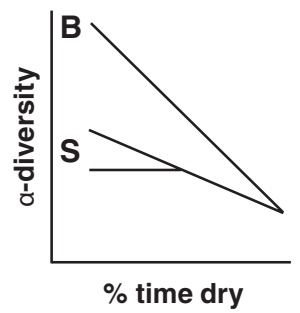

(b)

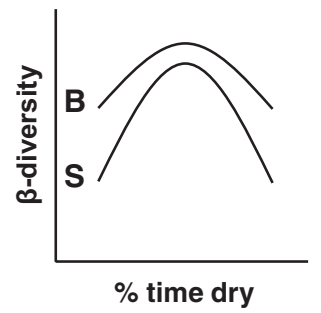

(c)

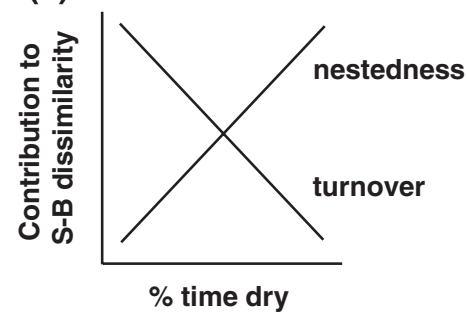

Figure 1. The direction and relative strength of hypothesized subsurface $(\mathrm{S})$ and benthic $(\mathrm{B})$ invertebrate community responses to water permanence (as the $\%$ of time a surface channel is dry): (a) change in $\alpha$-diversity, indicating two possible relationships for subsurface communities: a linear decrease or a threshold relationship; (b) change in $\beta$-diversity and (c) the relative contributions of nestedness (taxa gain or loss) and turnover (taxa replacement) to dissimilarity between subsurface and benthic communities. 


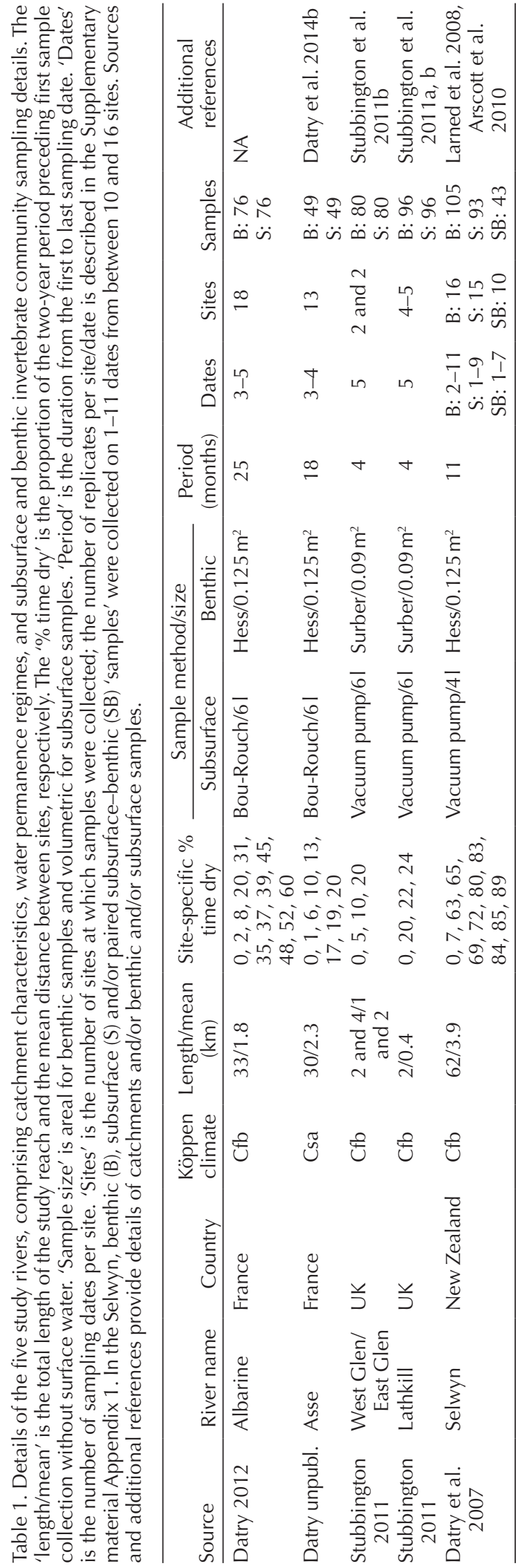

sometimes paired with multiple benthic samples and vice versa (Supplementary material Appendix 1). For each benthic, subsurface and paired subsurface-benthic data set, we calculated taxa richness (number of taxa sample ${ }^{-1}$ ) as a measure of $\alpha$-diversity. We assigned individuals of taxa identified to multiple taxonomic levels (e.g. to genus and species level) to the single most likely taxon, to avoid overestimating richness while maintaining the finest taxonomic resolution possible. The taxonomic resolution varied among data sets (Supplementary material Appendix 2 Table A1), enabling observation of species-specific responses which could have been obscured by a coarser common resolution. Combining and comparing data that reach different taxonomic resolutions is validated by strong correlations between species, genus and family-level responses in previous analyses (Heino and Soininen 2007, Datry et al. 2014a).

\section{Data analysis}

\section{Relationships between community composition and drying duration (hypothesis 1)}

To identify compositional differences and interactions between habitats (benthic, subsurface) and water permanence regimes (perennial, temporary) in each river, we ran permutational multivariate ANOVA (PERMANOVA; Anderson 2001) on Sørensen distance matrices calculated for paired subsurface-benthic samples, with presence-absence data accounting for differences in sampling methods between habitats. Where interactions were identified between habitat and permanence regime, we used PERMANOVA based on Bray-Curtis dissimilarity matrices of square-root-transformed abundance data to identify differences in subsurface community composition between perennial and temporary regimes. Where composition differed between regimes, we plotted non-metric multidimensional scaling (NMDS) ordinations of subsurface and, separately, benthic community composition in relation to $\%$ time dry. We built separate subsurface and benthic linear models (LM) to characterize relationships between NMDS dimension 1 and 2 values and \% time dry, and we report relationships identified as significant. We used analysis of covariance (ANCOVA) with habitat as a covariate to compare subsurface and benthic responses to drying, with the interaction term indicating whether response magnitude differed between communities.

\section{Relationships between $\alpha$-diversity and drying duration (hypothesis 2)}

We used LM to identify relationships between both benthic and, separately, subsurface richness and \% time dry for all sites on each individual river, and also for sites with $<25 \%$ time dry on all rivers (Table 1). The latter analysis enabled identification of general patterns across rivers and, specifically, explored the hypothesized biotic responses to shorter drying durations (i.e. greater water permanence; Fig. 1a). For the analysis of sites with $<25 \%$ time dry, we built separate subsurface and benthic all-river generalized linear mixed-effects 
models (GLMM) using a Poisson error distribution and a log link function. We tested the GLMM for overdispersion. We included river as a random factor. We also included method (e.g. Hess or Surber for benthic communities; Table 1) as a random factor in preliminary models, but identified no effect and hence excluded it from final models. We used model parameter estimates to calculate average rates of loss or gain of taxa in relation to \% time dry. Where both subsurface and benthic richness responded to drying duration, we used ANCOVA with habitat as a covariate to compare community response magnitudes.

\section{Comparison of subsurface and benthic $\beta$-diversity (hypothesis 3)}

We computed three distance matrices for separate subsurface and benthic communities in each individual river: total incidence-based $\beta$-diversity as the Sørensen index $\left(\beta_{\mathrm{SOR}}\right)$, and its nestedness-resultant $\left(\beta_{\mathrm{SNE}}\right)$ and turnover $\left(\beta_{\mathrm{SIM}}\right)$ components (Baselga and Orme 2012). $\beta_{\mathrm{SNE}}$ describes differences resulting from taxa gain or loss and $\beta_{\text {SIM }}$ indicates turnover, in which some taxa replace other taxa. We used a randomization-based permutational analysis of multivariate dispersion (PERMDISP; Anderson 2004, Oksanen et al. 2018) to identify differences in $\beta_{\mathrm{SOR}}$ and its components between subsurface and benthic communities.

\section{Relationships between $\beta$-diversity and drying duration (hypothesis 3)}

To explore relationships between mean $\beta$-diversity and \% time dry for separate subsurface and benthic communities, we calculated $\beta$-diversity matrices for each site (not each $\%$ time dry) on each individual river, to consistently quantify patterns at sites with both unique and shared drying durations. We used linear mixed-effects models (LMM) to assess relationships in an all-river analysis including sites with $<25 \%$ time dry, with random factors as described for hypothesis 2 . Where significant responses were identified for both subsurface and benthic communities, we used ANCOVA to test whether community responses magnitudes differed.

For rivers in which both $\alpha$-diversity and $\beta$-diversity responded to \% time dry, we used a null model to simulate random communities and thus assess if changes in $\beta$-diversity were driven by $\alpha$-diversity (Chase et al. 2011, Chase and Myers 2011). We used a quasiswap algorithm to conserve both original taxa frequencies and site-specific taxa richness (Miklós and Podani 2004). Where $\beta$-diversity differed from random expectation, patterns were taken to indicate deterministic factors (e.g. environmental filtering); where $\beta$-diversity did not differ from random expectation, we inferred that patterns reflected differences in $\alpha$-diversity. Observed mean site $\beta$-diversity was compared to $95 \%$ confidence intervals (CI) of null-model $\beta$-diversities derived from 999 randomly generated mean values for each site. We calculated deviations from the null expectation ( $\mathrm{z}$ values), expressed as the difference between the observed and expected site mean $\beta$-diversity divided by the standard deviation of the expected value for each site. We assessed the linear relationship between $\mathrm{z}$ values and $\%$ time dry to determine if changes in $\beta$-diversity were related to changes in community composition along water permanence gradients.

\section{Relationships between subsurface-benthic community dissimilarity and drying duration (hypothesis 4)}

To describe subsurface-benthic community dissimilarity, we calculated $\beta_{\mathrm{SOR}}$ and its $\beta_{\mathrm{SNE}}$ component for each pair of subsurface and benthic community samples. To complement $\beta_{\mathrm{SNE}}$ (the nestedness-resultant component of dissimilarity; Baselga 2012), we used NODF (Nestedness metric based on Overlap and Decreasing Fill; Almeida-Neto et al. 2008) to calculate true subsurface-benthic nestedness for each sample pair. To assess if differences between the observed and null NODF values were significant, we compared observed values to the $95 \%$ CI derived from 999 randomly generated values and expressed deviations from the null expectation as $\mathrm{z}$ values. We used LM to characterize relationships between $\%$ time dry and $\beta_{\mathrm{SOR}}, \beta_{\mathrm{SNE}}$ and NODF on all individual rivers except the Selwyn, for which we used LMM with pair as a random factor due to the non-independence of the multiple subsurface samples which shared one paired benthic sample and vice versa. We also used LMM to explore these relationships in an analysis of all sites with $<25 \%$ time dry, with random factors as described for hypothesis 2 .

\section{Taxon-specific responses to drying duration}

We used Threshold Indicator Taxa ANalysis (TITAN; Baker and King 2010) with 500 bootstrap replicates to identify changes in subsurface and benthic taxon occurrence and abundance in relation to water permanence. TITAN combines indicator value analysis (IndVal, Dufrêne and Legendre 1997) and change-point analysis to identify the association of each taxon present in $\geq 3$ samples with points along an environmental gradient, in this case water permanence. We identified instances in which a taxon that increased in the subsurface sediments in response to \% time dry experienced a concurrent decrease in benthic abundance, and also compared the $\%$ time dry thresholds for taxa identified by TITAN as declining in both habitats.

Analyses were done in $\mathrm{R}$ ver. 3.5.0 (R Core Team) using the packages betapart (Baselga and Orme 2012), blmeco (Korner-Nievergelt et al. 2019), lme4 (Bates et al. 2018), TITAN2 (Baker and King 2010) and vegan (Oksanen et al. 2018).

\section{Data deposition}

Data available from the Dryad Digital Repository: <http:// dx.doi.org/10.5061/dryad.84gr477> (Stubbington et al. 2019). 


\section{Results}

\section{Compositional community responses to drying duration (hypothesis 1)}

Community composition differed between subsurface and benthic habitats in all rivers and between perennial and temporary water permanence regimes in all rivers except the Asse (Supplementary material Appendix 2 Fig. A1, A2, Table A2). Interactions with habitat indicated contrasting subsurface and benthic community responses to water permanence in all rivers except the Albarine and Asse (Supplementary material Appendix 2 Fig. A1, A2, Table A2). Subsurface community composition also differed between perennial and temporary sites in all rivers except the Asse (PERMANOVA, $p=0.001$ ). Both subsurface and benthic community composition changed along the water permanence gradient in all rivers except the Asse (Supplementary material Appendix 2 Fig. A1, A2). Response magnitude was greater for benthic than subsurface communities in the Albarine (ANCOVA, $\left.\mathrm{F}_{1,148}=141.0, \mathrm{p}<0.001\right)$ and Glen $\left(\mathrm{F}_{1,154}=234.9, \mathrm{p}<0.001\right)$, greater for subsurface communities in the Lathkill $\left(\mathrm{F}_{1,188}=16.7, \mathrm{p}<0.001\right)$ and comparable for Selwyn communities $\left(\mathrm{F}_{1,196}=0.125, \mathrm{p}=0.724\right.$; Supplementary material Appendix 2 Fig. A1, A2).

\section{Responses of $\alpha$-diversity to drying duration (hypothesis 2)}

Supplementary material Appendix 2 Table A3-A5 report mean \pm SE taxa richness and abundance in subsurface and benthic communities. Across all sites with $<25 \%$ time dry, benthic richness decreased with increasing drying duration (GLMM, CI $[-0.007:<-0.001])$ whereas subsurface richness was stable (CI [-0.002:0.008]; Fig. 2a). Parameter estimates indicated that, on average, benthic taxa richness decreased by $3 \%$ with every $10 \%$ increase between 0 and $25 \%$ time dry (i.e. between 0 and $10 \%, 1$ and $11 \%$ etc.). Considering all sites on individual rivers, richness decreased with increasing drying duration for both communities in the Albarine and Selwyn, a moderate increase in subsurface richness occurred with increasing \% time dry in the Lathkill, and no trends were observed in the Asse or Glen (Fig. 2). ANCOVA indicated higher magnitude benthic compared to subsurface responses to drying duration, across sites on all rivers with $<25 \%$ time dry $\left(\mathrm{F}_{1,632}=63.0, \mathrm{p}<0.001\right)$, in the Albarine $\left(\mathrm{F}_{1,148}=36.5, \mathrm{p}<0.001\right)$ and in the Selwyn $\left(\mathrm{F}_{1,114}=26.6, \mathrm{p}<0.001\right.$; Fig. 2).

\section{Subsurface $\beta$-diversity and benthic $\beta$-diversity compared (hypothesis 3 )}

Across all rivers and both subsurface and benthic communities, total $\beta$-diversity $\left(\beta_{\mathrm{SOR}}\right)$ ranged between 0.39 and 0.62 (Supplementary material Appendix 2 Table A6). No pattern typified differences in $\beta$-diversity between subsurface and benthic communities (Supplementary material Appendix
2 Table A6): $\beta_{\text {SOR }}$ was higher in subsurface communities in the Glen and Lathkill, higher in benthic communities in the Selwyn, and comparably low in Albarine and Asse communities. $\beta_{\mathrm{SIM}}$ was consistently higher than $\beta_{\mathrm{SNE}}$ in both communities: variability among sites was driven more by taxa replacement than taxa loss or gain, with contrasting differences between communities observed across rivers for both components (Supplementary material Appendix 2 Fig. A3, Table A6).

\section{Responses of $\beta$-diversity to drying duration (hypothesis 3)}

In the all-river analysis, benthic $\beta_{\mathrm{SOR}}$ increased from 0 to $25 \%$ time dry (LMM CI [0.0005-0.0066]; Fig. 3a). In the Albarine, an increase in $\beta_{\mathrm{SOR}}$ with drying duration (Fig. 3b) reflected an increase in benthic community variability that was driven by declining $\alpha$-diversity (LM z value $\sim \%$ time dry, $\mathrm{t}=3.7, \mathrm{p}=0.002$; Fig. $2 \mathrm{~b}$ ): communities were more similar than expected by chance at low $\%$ time dry, but did not differ from random expectation as drying durations increased. The $\beta_{\mathrm{SOR}}$ of subsurface communities was stable across the water permanence gradient across all sites with $<25 \%$ time dry and in all individual rivers except the Selwyn (Supplementary material Appendix 2 Fig. A4). Here, $\beta_{\mathrm{SOR}}$ declined as drying duration increased, and changes in $\beta$-diversity (Fig. $3 \mathrm{~d}$ ) were independent of $\alpha$-diversity (LM $\mathrm{z}$ value $\sim \%$ time dry, $\mathrm{t}=0.86$, $\mathrm{p}=0.416$; Fig. 2f). No consistent, significant responses were identified for separate subsurface and benthic communities (Fig. 3, Supplementary material Appendix 2 Fig. A4), and so we did not compare response magnitudes.

\section{Responses of subsurface-benthic community dissimilarity to drying duration (hypothesis 4)}

No relationship was identified between \% time dry and subsurface-benthic community dissimilarity $\left(\beta_{\mathrm{SOR}}\right)$, across all sites with $<25 \%$ drying durations (LMM, CI [-0.002:0.001]; Supplementary material Appendix 2 Fig. A5a), or in any individual river except the Selwyn; here, $\beta_{\mathrm{SOR}}$ was high and increased with \% time dry (CI $[<0.001: 0.003]$; Supplementary material Appendix 2 Fig. A5f). Weak decreases in the $\beta_{\mathrm{SNE}}$ contribution to $\beta_{\text {SOR }}$ were identified with decreasing water permanence across all sites with $<25 \%$ drying durations (LMM CI $[-0.005:<-0.001])$ and in the Albarine and Selwyn (CI $[-0.002:<-0.001]$; Supplementary material Appendix 2 Fig. A6); no relationships with \% time dry were observed in other rivers. Comparable reductions in NODF values were observed with increasing drying duration in the Albarine and Selwyn (CI [-0.085:-0.030]); no relationship was identified across sites with $<25 \%$ time dry (CI [-0.030:0.018]; Supplementary material Appendix 2 Fig. A7).

\section{Taxon-specific responses to drying duration}

The subsurface occurrence of $\geq 1$ taxon increased in response to \% time dry in all rivers, with Cyclopoida (Copepoda) 

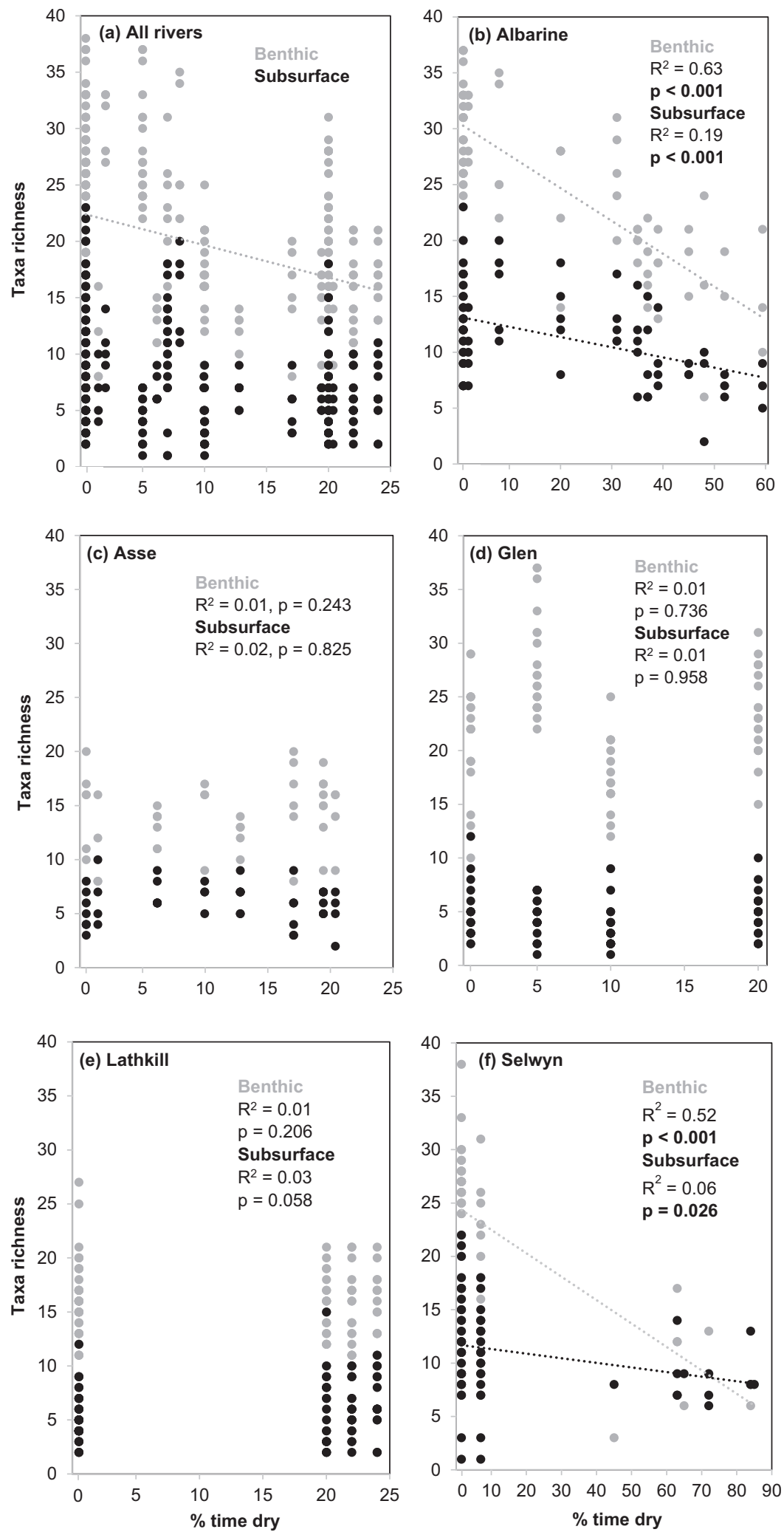

Figure 2. Subsurface and benthic invertebrate community $\alpha$-diversity (as taxonomic richness; taxa sample ${ }^{-1}$; sample sizes are in Table 1 ) in relation to the $\%$ of time without surface water in five rivers: (a) sites with $0-25 \%$ drying durations across all rivers; the (b) Albarine; (c) Asse; (d) Glen; (e) Lathkill and (f) Selwyn. Relationships identified using generalized linear mixed-effects models are described in the text for (a). 

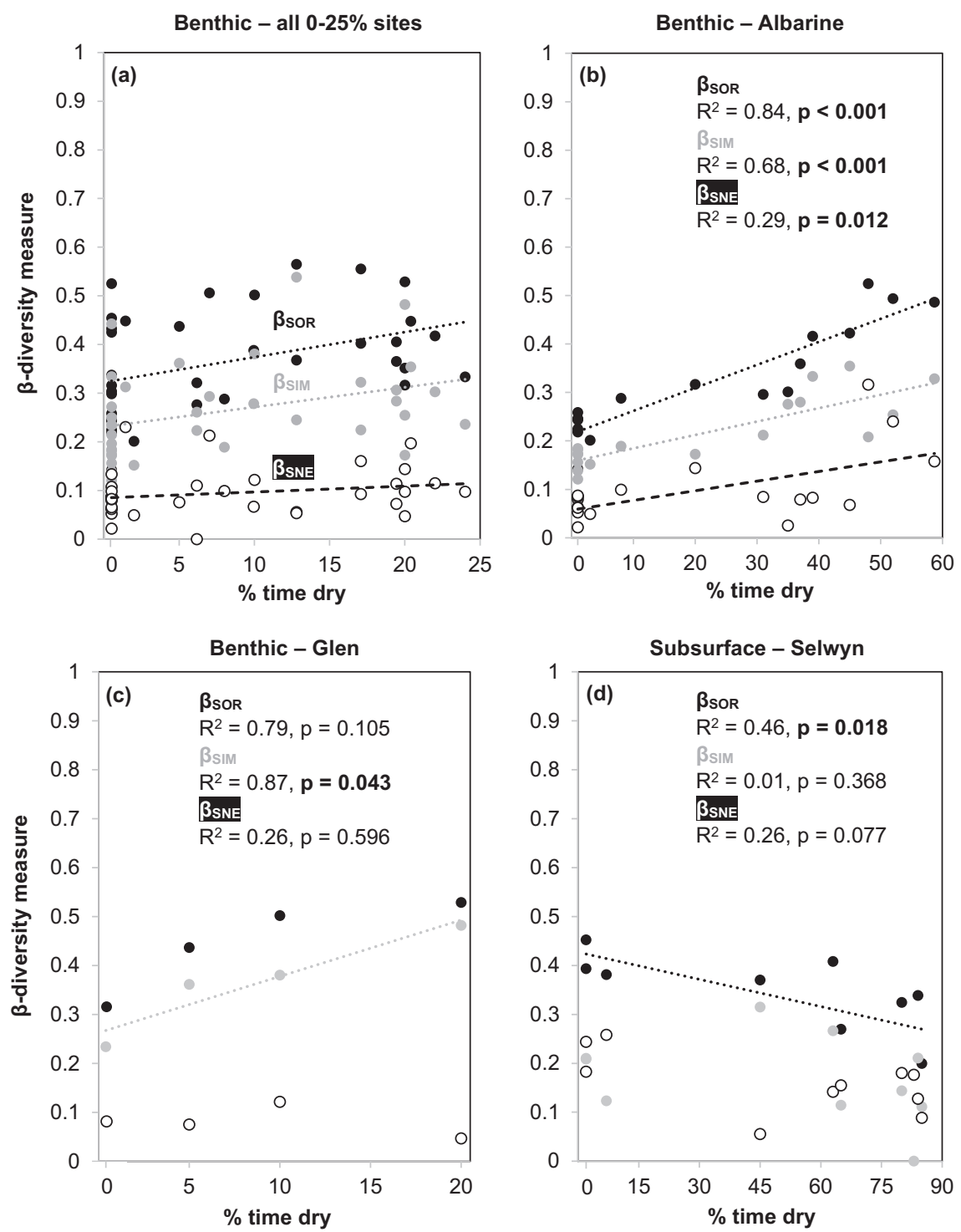

Figure 3. Invertebrate community $\beta$-diversity in relation to the $\%$ of time without surface water, based on the Sørensen index $\left(\beta_{\text {SOR }}\right)$ and its nestedness-resultant $\left(\beta_{\mathrm{SNE}}\right)$ and turnover $\left(\beta_{\mathrm{SIM}}\right)$ components. Benthic communities at sites: (a) with $0-25 \%$ drying durations across all rivers; (b) on the Albarine; (c) on the Glen and (d) subsurface communities on the Selwyn. Relationships identified using linear mixed-effects models are described in the text for (a).

showing high-magnitude responses at thresholds of $0-10 \%$ time dry in four rivers (Fig. 4). Other high-magnitude positive responses were observed for Gammarus pulex (Amphipoda) from 0 to $24 \%$ time dry and Ephemerella ignita (Ephemeroptera) and Ostracoda from 20 to $24 \%$ in the Lathkill, and Lumbriculus worms between 10 and $85 \%$ in the Selwyn (Fig. 4). Lower magnitude increases were recorded for the Chironomidae (Diptera) from 5 to $20 \%$ time dry in the Glen and 0 to $20 \%$ in the Lathkill, Caenis luctuosa (Ephemeroptera) from 5 to 20\% in the Glen, and Nemoura (Plecoptera) from 0 to $24 \%$ in the Lathkill (Fig. 4). A concurrent subsurface increase (Fig. 4) and benthic decline was not observed for any taxon, whereas concurrent increases occurred for chironomids from 5 to $20 \%$ time dry in the Glen (Supplementary material Appendix 2 Fig. A8). Thresholds at which a change began were identical for most taxa for which both subsurface (Fig. 4) and benthic (Supplementary material Appendix 2 Fig. A8) occurrence and abundance decreased.

\section{Discussion}

Recent research in disturbance-prone river ecosystems has emphasized the importance of lateral links among communities in supporting the integrity of wider metacommunities (Brown et al. 2011, Datry et al. 2016, Sarremejane et al. 2017). We highlight that extending 

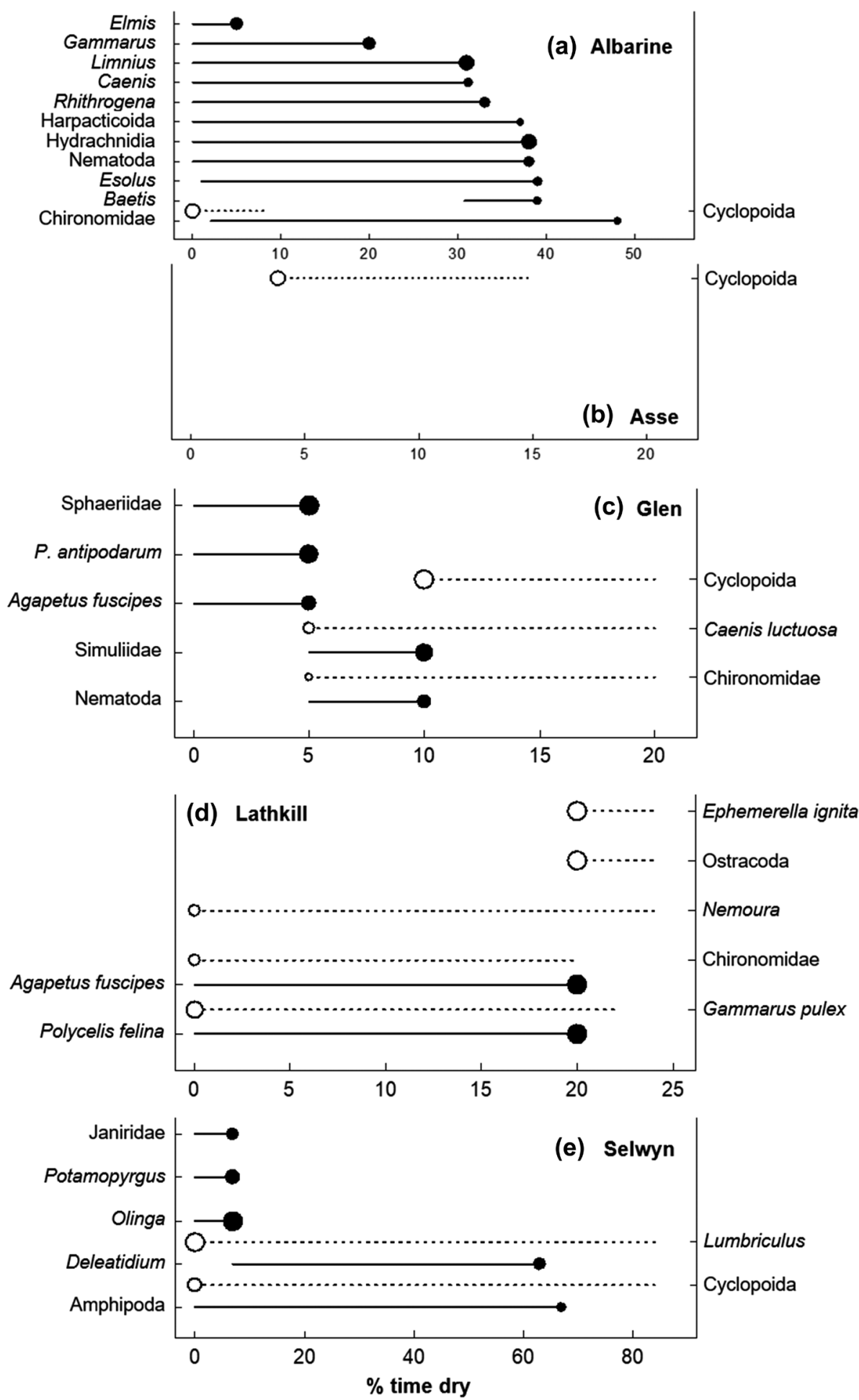

Figure 4. Threshold Indicator Taxa ANalysis (TITAN) plots of taxa whose subsurface occurrence decreased (black-filled circles, y axis 1) or increased (white-filled circles, y axis 2) in relation to the \% of time without surface water in five rivers: the (a) Albarine; (b) Asse; (c) Glen; (d) Lathkill and (e) Selwyn. Circle size indicates IndVal scores rescaled as z scores i.e. the relative magnitude of change in a taxon's frequency of occurrence. Horizontal lines extending from each symbol indicate the range within which change points occurred in $95 \%$ of bootstraps, with symbols indicating the earliest and latest change points for increases and decreases, respectively. Table 1 provides river-specific $\%$ time dry details, e.g. a threshold of $0 \%$ indicates responses at sites with $\geq 1,2,5,7$ and $20 \%$ time dry on the Asse, Albarine, Glen, Selwyn and Lathkill, respectively. 
ecological characterizations in the vertical dimension can further advance understanding of responses to disturbance, by recognizing changing dynamics within connected benthic-subsurface communities. Our results demonstrate the imprint of drying on instream communities and suggest it as a fundamental force that structures a community spanning the surface and subsurface sediments, with each contributor to this community making a distinct contribution to biodiversity.

\section{Effects of drying on subsurface and benthic community composition and $\alpha$-diversity}

Subsurface community composition changed but richness did not decline along water permanence gradients spanning short-to-moderate $(<25 \%)$ drying durations. These responses contrasted with those of benthic communities, which typically experienced greater compositional changes accompanied by reductions in richness. Subsurface interstices retain free water for longer, then maintain greater humidity compared to surface sediments (Valett et al. 1990), especially in temperate climates with year-round precipitation, as exemplified by our UK rivers (Table 1 ). These conditions can facilitate persistence of viable organisms within subsurface sediments until water availability falls below taxon-specific tolerance thresholds (Stanley et al. 1994, Clinton et al. 1996, Stubbington et al. 2009, Stubbington and Datry 2013). At sites experiencing longer ( $>25 \%$ ) dry periods, a decline in subsurface richness supported the threshold relationship for $\alpha$-diversity in our second hypothesis (Fig. 1a). Our results indicate that subsurface sediments can promote biotic persistence of low-abundance invertebrate communities in temporary rivers, but that their inhabitability typically declines as drying durations increase.

Permanent subsurface communities are often dominated by meiofauna, many species of which are desiccationtolerant and can thus survive dry phases within humid interstices (Supplementary material Appendix 2 Table A4, Clinton et al. 1996, Stubbington et al. 2017b). We recorded an increase in the subsurface occurrence of cyclopoid copepods as water permanence decreased in four of five rivers (Fig. 4), suggesting that drying may enable existing, adapted subsurface inhabitants to thrive in a low-predation niche if drying-sensitive competitors are lost (Dahms 1995, Stubbington et al. 2009, Milner et al. 2018). Positive biotic responses to declining permanence have previously been identified for adapted taxa in both benthic (Williams 1996, Bogan et al. 2013) and subsurface sediments (Datry et al. 2007). Our use of TITAN (Baker and King 2010) enabled the first quantitative comparison of taxon-specific subsurface and benthic change points on a water permanence gradient, thus enabling predictions of ecological responses to changing permanence regimes in a context of ongoing climate change (Costigan et al. 2016).

TITAN also identified water permanence thresholds at which the subsurface occurrence of primarily benthic taxa increased. Their migration into deeper sediments may have offset loss of desiccation-sensitive taxa, explaining the stable subsurface richness at shorter $(<25 \%)$ drying durations. Notably, the subsurface abundance of Gammarus pulex increased in the Lathkill above $0 \%$ time dry (Fig. 4d) while its benthic abundance remained stable (Supplementary material Appendix 2 Fig. A8c), indicating active migration into deeper sediments (Stubbington 2012). Stubbington et al. (2011a, b) suggest that declining submerged habitat availability in the Lathkill increased invertebrate densities, intensified intraspecific interactions, and thus triggered these vertical migrations (McGrath et al. 2007). In contrast, the subsurface population of $G$. pulex declined above $20 \%$ time dry in the Albarine (Fig. 4a), indicating that for this desiccationsensitive taxon, subsurface sediments became inhospitable as drying durations increased (Extence 1981, Stubbington et al. 2009, Vadher et al. 2018). Our results suggest that the refuge capacity of subsurface sediments changes over time during individual disturbances, as well as varying among events and among taxa (Dole-Olivier et al. 1997, Stubbington 2012, Vander Vorste et al. 2016).

\section{Subsurface $\beta$-diversity and benthic $\beta$-diversity compared}

Our hypothesis that $\beta$-diversity would be lower in subsurface compared to benthic communities (Fig. 1b) due to greater benthic habitat heterogeneity (Datry 2012) was only supported in the Selwyn (Supplementary material Appendix 2 Fig. A3, Table A6) and was driven by turnover. This likely reflects replacement of desiccation-sensitive taxa by drying-adapted taxa in response to benthic habitat variability along the Selwyn's $60-\mathrm{km}, 0-89 \%$ water permanence gradient, with a shift from communities dominated by caddisflies such as Olinga feredayi to those with high abundance of chironomids including Eukiefferiella (Arscott et al. 2010). In contrast to our hypothesis, $\beta$-diversity was higher for subsurface than benthic communities in the Lathkill and Glen. This may partly reflect inflated impacts of individual taxonomic differences in these taxa-poor communities (Chase 2007, Chase et al. 2011), their low richness likely reflecting collection using the vacuum-pump method (Stubbington et al. 2016) as well as the influence of anthropogenic activity on habitat quality (Stubbington 2011). Short among-site dispersal distances in these small catchments (Table 1) mean that dispersal by dominant taxa (i.e. Chironomidae in the Glen and G. pulex in the Lathkill; Supplementary material Appendix 2 Table A5) had greater potential to homogenize benthic communities than those in relatively inaccessible subsurface sediments (Heino et al. 2015). High subsurface $\beta$-diversity in the Lathkill also reflected the occurrence of primarily benthic taxa (e.g. G. pulex, Nemoura) in subsurface sediments at some sites, contributing to the changes in community composition and stable taxa richness observed at shorter drying durations. 


\section{Effects of drying on subsurface and benthic $\beta$-diversity}

Our hypothesized 'humped' response of $\beta$-diversity to drying duration (Fig. 1b) was informed by the theories that loss of drying-sensitive taxa (Chase 2007, Lepori and Malmqvist 2009) and mass effects (Heino et al. 2015) reduce variability in community composition at highly disturbed and undisturbed sites, respectively, whereas habitat heterogeneity enhances variability at sites with intermediate disturbance regimes ( Leigh and Datry 2017). We predicted greater changes for subsurface communities due to their fleeting supplementation by benthic migrants. Despite the described evidence for such migrations, we found no evidence of a humped relationship (informed by Leigh and Datry 2017) between $\beta$-diversity and water permanence (Fig. 1b) for either community.

Benthic $\beta$-diversity was stable (Supplementary material Appendix 2 Fig. A4) or increased linearly with declining water permanence, with positive associations identified across all sites with $<25 \%$ drying durations, and in the Albarine and Glen (Fig. 3). The increase in benthic $\beta$-diversity (and nestedness) with declining water permanence on the Albarine was driven by falling $\alpha$-diversity (Fig. 2b, Datry 2012): here, each taxonomic change had increasingly pronounced compositional effects in taxonpoorer communities as permanence declined, and no 'true' increase in compositional variability occurred (Chase et al. 2011). In contrast, the increase in $\beta$-diversity with drying duration across all sites with $0-25 \%$ time dry was independent of $\alpha$-diversity, suggesting that environmental filtering may not reduce $\beta$-diversity at lower disturbance intensities (Chase 2007). Instead, habitat heterogeneity, lower connectivity and longer-lasting priority effects may have augmented benthic $\beta$-diversity. The contrast with declines in $\beta$-diversity with increasing disturbance intensity in other freshwater ecosystems with predictable disturbance regimes (Lepori and Malmqvist 2009) may reflect greater environmental heterogeneity in rivers with temporary water permanence regimes. Here, changing community composition reflects shifts between taxa associated with flowing then ponded habitats as waters recede (Stubbington et al. 2017a, Tonkin et al. 2017) and community reassembly after flow resumes (Bogan and Lytle 2011, Sarremejane et al. 2017).

We only observed the hypothesized homogenizing effects of disturbance on subsurface $\beta$-diversity in the mobile sediments of the alluvial Selwyn (Larned et al. 2008), where lower diversities occurred at longer drying durations (Fig. 3d). The Selwyn's long (0-89\%) water permanence gradient indicates that environmental filtering and convergent niche selection influenced community composition at high disturbance intensities (Leigh and Datry 2017). Recolonization of temporary sites may be limited if distances to recolonist sources exceed those that even strong dispersers can achieve within short wet phases (Bogan et al. 2013), or if recruited juveniles are unable to complete their aquatic life stages before sites re-dry (Bogan and Lytle 2011). The contrast with benthic patterns may reflect the longer periods required for communities dominated by weak dispersers to recolonize relatively inaccessible subsurface sediments, with common subsurface inhabitants such as Olinga caddisflies and Deleatidium mayflies nearly absent from sites experiencing long dry durations. Stable subsurface $\beta$-diversity across the water permanence gradient in other rivers (Supplementary material Appendix 2 Fig. A4) contrasts with the linear increase in benthic $\beta$-diversity as drying increased. This stability may reflect the nature of subsurface habitats: their inaccessibility reduces community exposure to homogenizing mass effects by competitive taxa at more permanent sites, while their relative environmental homogeneity limits compositional changes compared to benthic communities. Stable subsurface $\beta$-diversity occurred despite the increasing subsurface occurrence of some primarily benthic taxa, indicating that subsurface community structure is resistant to such short-term 'invasions'.

\section{Effects of drying on subsurface-benthic dissimilarity}

Despite the increasing subsurface occurrence of benthic taxa, we found no evidence for the hypothesized decrease in dissimilarity between subsurface and benthic communities or increasing contribution of nestedness to dissimilarity as drying durations increased (Fig. 1c). Dissimilarity and water permanence were only related in the Selwyn, where distinct subsurface and benthic communities (Supplementary material Appendix 2 Table A3-A5) became more dissimilar with drying duration (Supplementary material Appendix 2 Fig. A5f). Subsurface-benthic nestedness declined as permanence decreased in the Albarine, Selwyn and across all sites with $<25 \%$ time dry (Supplementary material Appendix 2 Fig. A6). Our hypotheses were informed by evidence that the subsurface sediments can be a drying refuge (Stubbington 2012, Vander Vorste et al. 2016), but local habitat conditions such as rapid subsurface water loss after surface drying may have prevented persistence of predominantly benthic taxa within interstitial spaces in the Selwyn (Datry et al. 2007) and Albarine (Datry 2012). Our results suggest that subsurface sediments may be inhabited by communities that are resistant to influxes of colonists and that - despite lower macroinvertebrate $\alpha$-diversity - have their own distinct identity (PeraltaMaraver et al. 2018), their distinctness increasing as water permanence declines.

\section{Conclusions}

We extend understanding of how local $\alpha$-diversity and its spatial organization ( $\beta$-diversity) respond to disturbance within highly connected contributors to wider metacommunities that inhabit three-dimensional landscapes. We suggest that 
communities within subsurface habitats contribute to the biodiversity of metacommunities connected by dispersal in both horizontal and vertical planes. Subsurface communities are exposed to relatively limited environmental variability, and thus support a diverse, functionally important biota during disturbances. By highlighting that vertical connectivity can promote community persistence during disturbance, our results inform the development of effective monitoring and management strategies that underpin the ecological resilience of dynamic ecosystems in a changing world (Socolar et al. 2016). We observed both common and contrasting patterns in temperate-zone rivers across two continents. Contrasting patterns partly reflect our inclusion of data collected throughout hydrological cycles, which enabled identification of general relationships between environmental drivers and community responses irrespective of temporal variability. Future research could build on our work by exploring global and contextdependent patterns of temporal variability in subsurfacebenthic $\alpha$ and $\beta$-diversity responses to drying disturbances.

Acknowledgements - We thank Scott Larned for generous provision of the River Selwyn data, and Richard Chadd/Jenni Murphy and John Gunn for describing the water permanence regime of the Rivers Glen and Lathkill, respectively. We thank Jani Heino, three anonymous reviewers and the editor whose insightful comments greatly enhanced earlier versions of our manuscript.

Funding - RSt was supported by a grant for a short-term scientific mission to IRSTEA Research Inst., France, within the COST Action CA15113 (SMIRES, Science and Management of Intermittent Rivers and Ephemeral Streams, <www.smires. eu>), by a Nottingham Trent Univ. (NTU) 'Sustainable Futures Research Theme' travel grant, and by an NTU School of Animal, Rural and Environmental Sciences international collaboration grant. TD received funds supporting this research from the Rhône-Mediterranée-Corse Water Agency and IRSTEA.

\section{References}

Almeida-Neto, M. et al. 2008. A consistent metric for nestedness analysis in ecological systems: reconciling concept and measurement. - Oikos 117: 1227-1239.

Anderson, M. J. 2001. A new method for non-parametric multivariate analysis of variance. - Austral Ecol. 26: 32-46.

Anderson, M. J. 2004. PERMDISP: a FORTRAN computer program for permutational analysis of multivariate dispersions (for any two-factor ANOVA design) using permutation tests. - Dept of Statistics, Univ. of Auckland, New Zealand, p. 24.

Anderson, M. J. et al. 2011. Navigating the multiple meanings of $\beta$ diversity: a roadmap for the practicing ecologist. - Ecol. Lett. 14: 19-28.

Arscott, D. B. et al. 2010. Aquatic invertebrate community structure along an intermittence gradient: Selwyn River, New Zealand. - J. N. Am. Benthol. Soc. 29: 530-545.

Aspin, T. W. et al. 2018. Drought intensification drives turnover of structure and function in stream invertebrate communities. - Ecography 41: 1992-2004.
Baker, M. E. and King, R. S. 2010. A new method for detecting and interpreting biodiversity and ecological community thresholds. - Methods Ecol. Evol. 1: 25-37.

Baselga, A. 2010. Partitioning the turnover and nestedness components of beta diversity. - Global Ecol. Biogeogr. 19: 134-143.

Baselga, A. and Orme, C. D. L. 2012. Betapart: an R package for the study of beta diversity. - Methods Ecol. Evol. 3: 808-812.

Bates, D. et al. 2018. Lme4: linear mixed-effects models using 'Eigen' and S4, ver. 1.1-19. - < https://cran.r-project.org/web/ packages/lme4/index.html $>$.

Berryman, A. A. et al. 2006. The refuge as an integrating concept in ecology and evolution. - Oikos 115: 192-196.

Bogan, M. T. and Lytle, D. A. 2011. Severe drought drives novel community trajectories in desert stream pools. - Freshwater Biol. 56: 2070-2081.

Bogan, M. T. et al. 2013. Flow intermittency alters longitudinal patterns of invertebrate diversity and assemblage composition in an arid-land stream network. - Freshwater Biol. 58: 1016-1028.

Brown, B. L. et al. 2011. Metacommunity theory as a multispecies, multiscale framework for studying the influence of river network structure on riverine communities and ecosystems. - J. N. Am. Benthol. Soc. 30: 310-327.

Cadotte, M. W. 2007. Competition-colonization trade-offs and disturbance effects at multiple scales. - Ecology 88: 823-829.

Chase, J. M. 2007. Drought mediates the importance of stochastic community assembly. - Proc. Natl Acad. Sci. USA 104: $17430-17434$.

Chase, J. M. and Myers, J. A. 2011. Disentangling the importance of ecological niches from stochastic processes across scales. - Phil. Trans. R. Soc. B 366: 2351-2363.

Chase, J. M. et al. 2011. Using null models to disentangle variation in community dissimilarity from variation in $\alpha$-diversity. - Ecosphere 2: 1-11.

Clinton, S. M. et al. 1996. Response of a hyporheic invertebrate assemblage to drying disturbance in a desert stream. - J. N. Am. Benthol. Soc. 15: 700-712.

Costigan, K. H. et al. 2016. Understanding controls on flow permanence in intermittent rivers to aid ecological research: integrating meteorology, geology and land cover. - Ecohydrology 9: 1141-1153.

Dahms, H. 1995. Dormancy in the Copepoda - an overview. - Hydrobiologia 306: 199-211.

Datry, T. 2012. Benthic and hyporheic invertebrate assemblages along a flow intermittence gradient: effects of duration of dry events. - Freshwater Biol. 57: 563-574.

Datry, T. et al. 2007. Responses of hyporheic invertebrate assemblages to large-scale variation in flow permanence and surface-subsurface exchange. - Freshwater Biol. 52: 1452-1462.

Datry, T. et al. 2014a. Broad-scale patterns of invertebrate richness and community composition in temporary rivers: effects of flow intermittence. - Ecography 37: 94-104.

Datry, T. et al. 2014b. Ground-dwelling arthropod communities across braided river landscape mosaics: a Mediterranean perspective. - Freshwater Biol. 59: 1308-1322.

Datry, T. et al. 2016. Towards understanding the organisation of metacommunities in highly dynamic ecological systems. - Oikos 125: 149-159. 
Davis, J. et al. 2013. Evolutionary refugia and ecological refuges: key concepts for conserving Australian arid zone freshwater biodiversity under climate change. - Global Change Biol. 19: 1970-1984.

Denslow, J. S. 1980. Patterns of plant species diversity during succession under different disturbance regimes. - Oecologia 46: $18-21$.

Dole-Olivier, M. J. et al. 1997. Response of invertebrates to lotic disturbance: is the hyporheic zone a patchy refugium? - Freshwater Biol. 37: 257-276.

Dufrêne, M. and Legendre, P. 1997. Species assemblages and indicator species: the need for a flexible asymmetrical approach. - Ecol. Monogr. 67: 345-366.

Extence, C. A. 1981. The effect of drought on benthic invertebrate communities in a lowland river. - Hydrobiologia 83: 217-224.

Fernández-Juricic, E. 2002. Can human disturbance promote nestedness? A case study with breeding birds in urban habitat fragments. - Oecologia 131: 269-278.

Grime, J. P. 1973. Competitive exclusion in herbaceous vegetation. - Nature 242: 344-347.

Heino, J. and Soininen, J. 2007. Are higher taxa adequate surrogates for species-level assemblage patterns and species richness in stream organisms? - Biol. Conserv. 137: 78-89.

Heino, J. et al. 2015. Metacommunity organisation, spatial extent and dispersal in aquatic systems: patterns, processes and prospects. - Freshwater Biol. 60: 845-869.

Huston, M. 1979. A general hypothesis of species diversity. - Am. Nat. 113: 81-101.

Korner-Nievergelt, F. et al. 2019. Package 'blmeco', ver. 1.3. - $\quad<$ https://cran.r-project.org/web/packages/blmeco/index. html>.

Larned, S. T. et al. 2008. The Selwyn River of New Zealand: a benchmark system for alluvial plain rivers. - River Res. Appl. 24: $1-21$

Leigh, C. and Datry, T. 2017. Drying as a primary hydrological determinant of biodiversity in river systems: a broad-scale analysis. - Ecography 40: 487-499.

Lepori, F. and Malmqvist, B. 2009. Deterministic control on community assembly peaks at intermediate levels of disturbance. - Oikos 118: 471-479.

McGrath, K. E. et al. 2007. Habitat-mediated cannibalism and microhabitat restriction in the stream invertebrate Gammarus pulex. - Hydrobiologia 589: 155-164.

Met Office. 2019. Climate summaries. Met Office, Exeter, UK. - <https://www.metoffice.gov.uk/research/climate/maps-anddata/summaries/index $>$.

Miklós, I. and Podani, J. 2004. Randomization of presence-absence matrices: comments and new algorithms. - Ecology 85: 86-92.

Milner, A. M. et al. 2018. River ecosystem resilience to extreme flood events. - Ecol. Evol. 8: 8354-8363.

Oksanen, J. et al. 2018. Vegan: community ecology package, ver. $2.5-3 .-<$ http://CRAN.R-project.org/package=vegan $>$.

Peralta-Maraver, I. et al. 2018. Environmental filtering and community delineation in the streambed ecotone. - Sci. Rep. 8: 15871 .

Pickett, S. T. A. et al. 1989. The ecological concept of disturbance and its expression at various hierarchical levels. - Oikos 54: 129-136.

Sarremejane, R. et al. 2017. Do metacommunities vary through time? Intermittent rivers as model systems. - J. Biogeogr. 44: 2752-2763.
Socolar, J. B. et al. 2016. How should beta-diversity inform biodiversity conservation? - Trends Ecol. Evol. 31: 67-80.

Stanley, E. H. et al. 1994. Invertebrate resistance and resilience to intermittency in a desert stream. - Am. Midl. Nat. 131: 288-300.

Stubbington, R. 2011. The hyporheic zone as a refugium for benthic invertebrates in groundwater-dominated streams. - PhD thesis, Loughborough Univ., UK, p. 354.

Stubbington, R. 2012. The hyporheic zone as an invertebrate refuge: a review of variability in space, time, taxa and behaviour. - Mar. Freshwater Res. 63: 293-311.

Stubbington, R. and Datry, T. 2013. The macroinvertebrate seedbank promotes community persistence in temporary rivers across climate zones. - Freshwater Biol. 58: 1202-1220.

Stubbington, R. et al. 2009. The response of perennial and temporary headwater stream invertebrate communities to hydrological extremes. - Hydrobiologia 630: 299-312.

Stubbington, R. et al. 2011a. Benthic and hyporheic invertebrate community responses to seasonal flow recession in a groundwater-dominated stream. - Ecohydrology 4: 500-511.

Stubbington, R. et al. 2011b. Spatial variability in the hyporheic zone refugium of temporary streams. - Aquat. Sci. 73: 499-511.

Stubbington, R. et al. 2016. Characterization of macroinvertebrate communities in the hyporheic zone of river ecosystems reflects the pump-sampling technique used. - PLoS One 11: e0164372.

Stubbington, R. et al. 2017a. Temporary streams in temperate zones: recognizing, monitoring and restoring transitional aquatic-terrestrial ecosystems. - Wiley Interdiscip. Rev. Water 4: e1223.

Stubbington, R. et al. 2017b. The biota of intermittent rivers and ephemeral streams: aquatic invertebrates. - In: Datry, T. et al. (eds), Intermittent rivers and ephemeral streams: ecology and management. Elsevier, pp. 217-243.

Stubbington, R. et al. 2019. Data from: Alpha and beta diversity of connected benthic-subsurface invertebrate communities respond to drying in dynamic river ecosystems. - Dryad Digital Repository, <http://dx.doi.org/10.5061/dryad.84gr477>.

Szewczyk, T. M. and McCain, C. M. 2019. Disentangling elevational richness: a multi-scale hierarchical Bayesian occupancy model of Colorado ant communities. - Ecography 42: 977-988.

Tonkin, J. D. et al. 2017. Seasonality and predictability shape temporal species diversity. - Ecology 98: 1201-1216.

Townsend, C. R. 1989. The patch dynamics concept of stream community ecology. - J. N. Am. Benthol. Soc. 8: 36-50.

Tuomisto, H. 2010. A diversity of beta diversities: straightening up a concept gone awry. Part 1 . Defining beta diversity as a function of alpha and gamma diversity. - Ecography 33: 2-22.

Vadher, A. N. et al. 2018. The duration of channel drying affects survival of Gammarus pulex (Amphipoda: Gammaridae) within subsurface sediments: an experimental flume study. - Hydrobiologia 820: 165-173.

Valett, H. M. et al. 1990. Physical and chemical characteristics of the hyporheic zone of a Sonoran Desert stream. - J. N. Am. Benthol. Soc. 9: 201-215.

Vander Vorste, R. et al. 2016. Is drift the primary process promoting the resilience of river invertebrate communities? A manipulative field experiment in an intermittent alluvial river. - Freshwater Biol. 61: 1276-1292. 
Vanschoenwinkel, B. et al. 2010. Species sorting in space and time - the impact of disturbance regime on community assembly in a temporary pool metacommunity. - J. N. Am. Benthol. Soc. 29: 1267-1278.

Wesche, K. et al. 2000. The significance of fire for afroalpine ericaceous vegetation. - Mt. Res. Dev. 20: 340-348.

Supplementary material (available online as Appendix ecog04592 at <www.ecography.org/appendix/ecog-04592>). Appendix 1-2.
Williams, D. D. 1996. Environmental constraints in temporary fresh waters and their consequences for the insect fauna. - J. N. Am. Benthol. Soc. 15: 634-650.

Young, B. A. et al. 2011. Is the hyporheic zone a refuge for macroinvertebrates in drying perennial streams? - Mar. Freshwater Res. 62: 1373-1382. 